\title{
Clinical practice and implementation of enhanced recovery programs in hepato-pancreato-biliary surgery
}

\author{
Liming Chen, Lixia Cai, Beilei Gao, Xiangying Zhang \\ Division of Hepatobiliary Pancreatic Surgery, The First Affiliated Hospital, Zhejiang University School of Medicine, Hangzhou 310003, China \\ Correspondence to: Liming Chen. Division of Hepatobiliary Pancreatic Surgery, The First Affiliated Hospital, Zhejiang University School of Medicine, \\ 79 Qingchun Road, Hangzhou 310003, China. Email: 1192010@zju.edu.cn.
}

Submitted Aug 31, 2018. Accepted for publication Sep 01, 2018.

doi: 10.21037/hbsn.2018.09.01

View this article at: http://dx.doi.org/10.21037/hbsn.2018.09.01

The concept of enhanced recovery after surgery (ERAS) is developed according to the evidence-based medicine. ERAS reduces the physiological and psychological stress in surgical patients. It aims at expediting postoperative recovery by implementing specific strategies in perioperative management. It optimizes the perioperative care through the multidisciplinary collaboration of surgeons, anesthesiologists, nurses and nutritionists; reduces the perioperative stress response and postoperative complications; shortens the hospital stay, and promotes the patient rehabilitation (1).

The concept of ERAS was first proposed by a Danish surgeon Kehlet in 1997 (2). ERAS has been gradually applied to various fields of the global surgical world since then $(3,4)$. After 20 years of clinical practice, ERAS has been proved to reduce the total hospitalization expenditure, shorten the duration of hospitalization, reduce the incidence of postoperative complications, and improve the quality of healthcare services $(5,6)$. Thus, high awareness on policy level is recognized by increasingly numbers of countries in terms of developing protocols in ERAS. ERAS has become a government-led clinical pathway in Europe and the united states. The concept of ERAS was introduced to china in 2007. After more than a decade application, it has attracted great attention from academic scholars in Chinese healthcare society. It has been now applied in tertiary hospitals with solid professional strength and great technical support $(7,8)$.

The implications of ERAS include a variety of core elements. Firstly, it must include preoperative patient education. Secondly, the bowel preparation is not applied as a preoperative routine procedure, but is selectively applied to patients requiring colorectal surgery. Thirdly, it shortens the duration of preoperative fasting and optimizes anesthesia process. Fourthly, it should include the proper use of surgical minimally invasive techniques and avoid routine use of nasogastric tubes and intraoperative hypothermia. Moreover, it should include the restriction on fluid infusion, active treatment of postoperative pain, nausea and vomiting. Lastly, it should include the encouragement towards exercise early after surgery and start enteral intake as soon as they could. This optimized clinical pathway runs complete treatment processes through patients' preadmission, preoperative, intraoperative, postoperative, and post-discharge, with the core value of emphasizing the concept of providing patient-centered care.

In addition, by considering the complexity of the diagnosis and treatment of hepatic and pancreatic diseases, preoperative routine process should include a collaboration of medical imaging, endoscopy, pathology, oncology, radiotherapy, gastrointestinal, anesthesia and physicians from other related specialties to form a multidisciplinary team (MDT), to discuss clinical problems in terms of the diagnosis, further improvement of examination, indications for surgery, resectability assessment, preoperative neoadjuvant therapy, and treatment of comorbidities, to develop individualized treatment plans. Therefore, the ERAS concept emphasizes the multidisciplinary collaboration, which is the premise and basis for ERAS.

At present, the ERAS society has issued a series of ERAS clinical practice guidelines for liver surgery, pancreaticoduodenectomy $(9,10)$. Recently, some ERAS expert consensus or clinical pathway management guidelines have also been published in china $(11,12)$. 


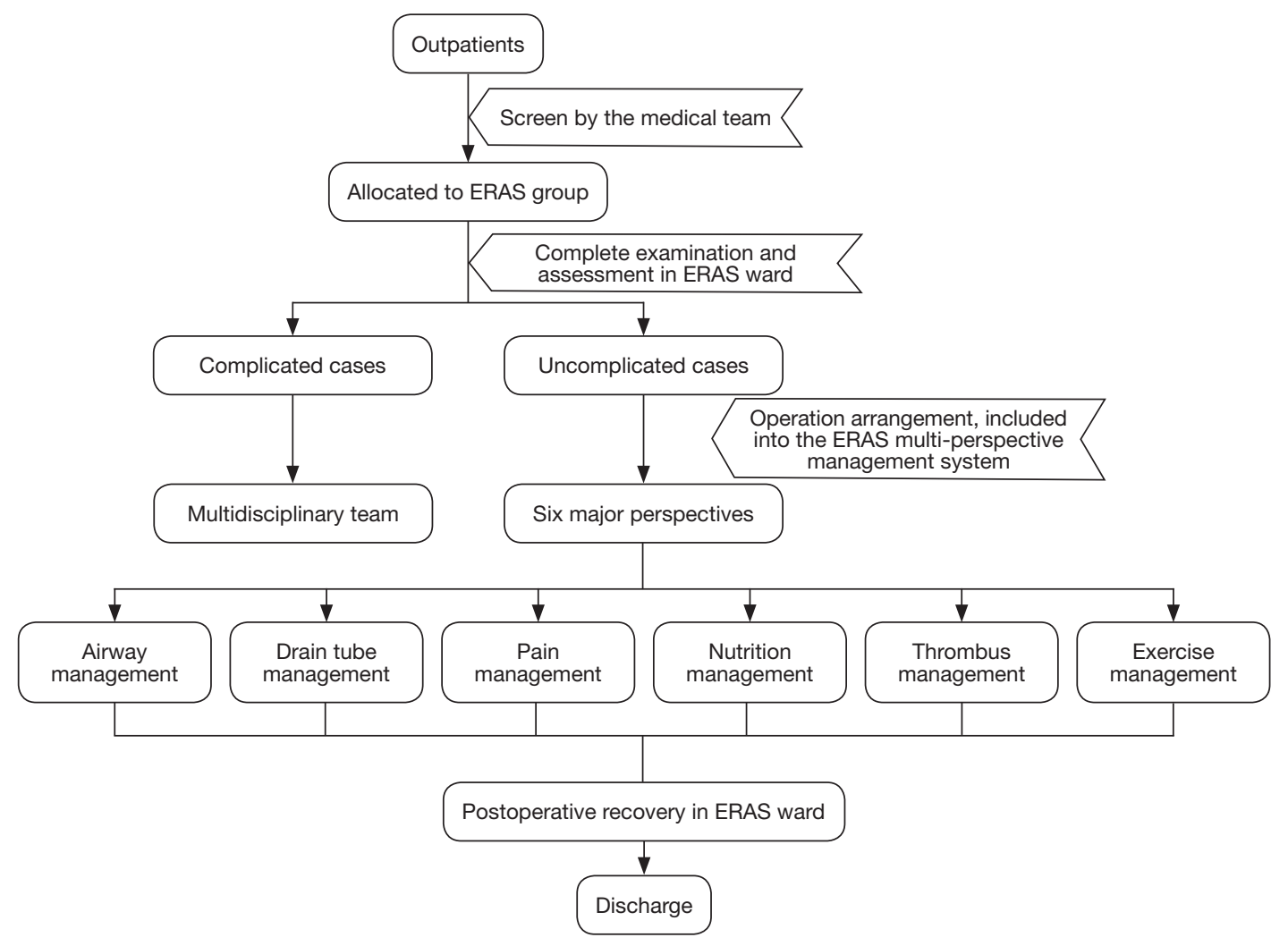

Figure 1 ERAS implementation standardization process in our hospital. ERAS, enhanced recovery after surgery.

These ERAS guidelines or pathways are derived from clinical practice and have been developed under evidencebased medical optimization of previous perioperative treatment interventions with support by high level of evidence. However, because of the complexity of clinical practice and the individual variation of patients, the implementation of ERAS cannot be generalized. The multifactorial considerations, such as patient condition, the treatment process, the department and the hospital facilities are necessary. In addition, there are still many areas of clinical practice that lack sufficient clinical based evidence and require more clinical studies to validate. For example, in hepatectomy, there is still insufficient clinical evidence in the management of patients with cirrhosis and hepatic insufficiency. The study on largescale hepatectomy, perioperative fluid therapy, and the impact of precise hepatectomy are needed on ERAS. In our hospital, the application of ERAS for hepatobiliary and pancreatic diseases has been carried out for more than 4 years, and achieved good clinical results and rich clinical experience. We have developed a unique ERAS multi- perspective management system and ERAS implementation standardization process (Figure 1). The core value of this system is on patient airway management and covers six major perspectives: airway management, drain tube management, pain management, nutrition management, thrombus management and exercise rehabilitation.

Overall, given the highly effective clinical practice of the ERAS and the high recognition and increased attention of the medical society in china and worldwide, the future development trend of ERAS is to be applied routinely in hospital surgical practice and clinical pathway. The scope of practice will also gradually expand from the current elective, single and typical surgery to emergency, severe trauma, infection and other critical illnesses. It must be emphasized that during the ERAS process, attention should be paid to shortening the hospitalization, reducing medical expenses, and reducing the rate of re-admission. Adhering to the basic principles of safety first and efficiency second, standardizing medical behavior, strengthening professional communication and constructing a safety management system during the operation period will enable ERAS to be 
carried out and implemented more safely and effectively.

\section{Acknowledgements}

Funding: This study was supported by a grant from the Project of Health and Family Planning Commission of Zhejiang province (NO.2018ZD004).

\section{Footnote}

Conflicts of Interest: The authors have no conflicts of interest to declare.

\section{References}

1. Ljungqvist $O$, Scott $M$, Fearon KC. Enhanced Recovery After Surgery: A Review. JAMA Surg 2017;152:292-8.

2. Kehlet H. Multimodal approach to control postoperative pathophysiology and rehabilitation. Br J Anaesth 1997;78:606-17.

3. Zouros E, Liakakos T, Machairas A, et al. Improvement of gastric emptying by enhanced recovery after pancreaticoduodenectomy. Hepatobiliary Pancreat Dis Int 2016;15:198-208.

4. Abraham N, Albayati S. Enhanced recovery after surgery programs hasten recovery after colorectal resections. World J Gastrointest Surg 2011;3:1-6.

5. Grant MC, Yang D, Wu CL, et al. Impact of Enhanced Recovery After Surgery and Fast Track Surgery Pathways on Healthcare-associated Infections: Results From

Cite this article as: Chen L, Cai L, Gao B, Zhang X. Clinical practice and implementation of enhanced recovery programs in hepato-pancreato-biliary surgery. HepatoBiliary Surg Nutr 2018;7(6):492-494. doi: 10.21037/hbsn.2018.09.01 a Systematic Review and Meta-analysis. Ann Surg 2017;265:68-79. Erratum in: Ann Surg 2017;266:e123.

6. Stone $A B$, Grant $M C$, Pio Roda $C$, et al. Implementation Costs of an Enhanced Recovery After Surgery Program in the United States: A Financial Model and Sensitivity Analysis Based on Experiences at a Quaternary Academic Medical Center. J Am Coll Surg 2016;222:219-25.

7. Rao JH, Zhang F, Lu H, et al. Effects of multimodal fast-track surgery on liver transplantation outcomes. Hepatobiliary Pancreat Dis Int 2017;16:364-9.

8. Dai J, Jiang Y, Fu D. Reducing postoperative complications and improving clinical outcome: Enhanced recovery after surgery in pancreaticoduodenectomy - A retrospective cohort study. Int J Surg 2017;39:176-81.

9. Melloul E, Hübner M, Scott M, et al. Guidelines for Perioperative Care for Liver Surgery: Enhanced Recovery After Surgery (ERAS) Society Recommendations. World J Surg 2016;40:2425-40.

10. Lassen K, Coolsen MM, Slim K, et al. Guidelines for perioperative care for pancreaticoduodenectomy: Enhanced Recovery After Surgery (ERAS®) Society recommendations. Clin Nutr 2012;31:817-30.

11. Jia W, Liu W, Qiao X. Chinese Expert Consensus on Enhanced Recovery After Hepatectomy (Version 2017). Asian J Surg 2018. [Epub ahead of print].

12. Chinese Research Hospitals Association, Committee of Enhanced Recovery After Surgery; China Ambulatory Surgery Alliance. Expert consensus on standardized processes of ambulatory biliary surgery (2018 edition). Zhonghua Wai Ke Za Zhi 2018;56:321-7. 